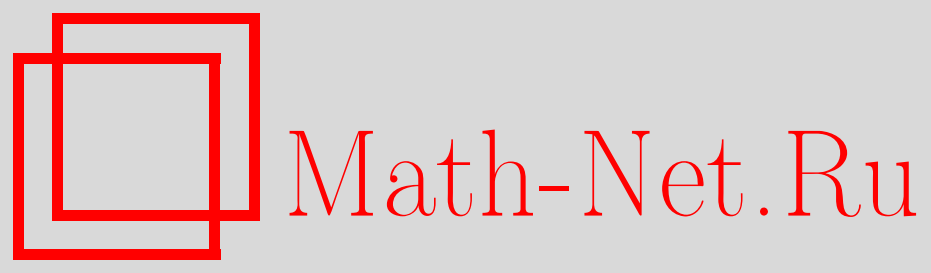

В. Брагин, Ряд чисел, обратных к простым, Квант, 2019, номер 5, 30-33

DOI: https://doi.org/10.4213/kvant20190503

Использование Общероссийского математического портала Math-Net.Ru подразумевает, что вы прочитали и согласны с пользовательским соглашением http://www.mathnet.ru/rus/agreement

Параметры загрузки:

IP : 3.85 .7 .115

26 апреля 2023 г., $17: 22: 34$

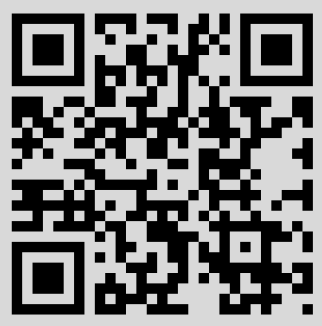


денсаторе, равная амплитуде ЭДС самоиндукции. Для того чтобы зажигалась искра, необходимо выполнение условия $U_{0}>900$ В. Попробуем определить $U_{0}$, используя закон сохранения энергии. К моменту достижения максимального напряжения на конденсаторе $\tilde{\varnothing}+U_{0}$ ток станет равен нулю, а ЭДС переместит заряд $C\left(\delta+U_{0}\right)$ и совершит работу $C \delta\left(\delta+U_{0}\right)$, которая равна суммарному изменению энергии катушки и конденсатора. Эти соображения количественно отражает соотношение

$$
C \bar{\varnothing}\left(\tilde{\xi}+U_{0}\right)=\frac{C\left(\tilde{\delta}+U_{0}\right)^{2}}{2}-\frac{L I_{0}^{2}}{2} .
$$

После преобразований получим

$$
U_{0}^{2}=\varnothing^{2}+\frac{L}{C} I_{0}^{2} \text {, и } U_{0}=\varnothing \sqrt{1+\frac{0,81 L}{R^{2} C}} .
$$

\section{Ряд чисел, обратных к простым}

\section{В.БРАГИН}

В математике часто в том или ином виде встречается понятие бесконечности. Например, рассматриваются суммы с неограниченным количеством слагаемых (на самом деле в статье-то почти все суммы конечны) и бесконечные суммы, называемые числовыми рядами. В вопросах о бесконечных множествах опыт работы с конечными объектами иногда абсолютно не помогает. Преподавая у детей в кружках, всегда интересно наблюдать за первыми встречами школьников с бесконечными суммами. Видно, как трудно дается освоение этого нового мира со своими законами.

Начнем со следующей классической теоремы про так называемый гармонический ряд, или ряд из обратных к натуральным числам.

Теорема 1. Сумма $1+\frac{1}{2}+\frac{1}{3}+\frac{1}{4}+\ldots+\frac{1}{N}$ может быть больше любого наперед заданного числа.

Доказательство. Заметим, что

$$
\frac{1}{3}+\frac{1}{4}>\frac{1}{2}, \frac{1}{5}+\frac{1}{6}+\frac{1}{7}+\frac{1}{8}>\frac{1}{2},
$$

DOI: https://doi.org/10.4213/kvant20190503
Напомним, что $\delta=n \varnothing_{0}$, где $n$ - количество аккумуляторов, $\tilde{\varnothing}_{0}-$ ЭДС одного аккумулятора, $R=n r_{0}+R_{0}-$ общее сопротивление в цепи первичной обмотки. Прикинем теперь, сколько нужно аккумуляторов, чтобы искровой промежуток пробивался. Если $n=1$, то амплитуда напряжения равна

$$
U_{0}^{(1)}=12 \sqrt{1+\frac{0,81 \cdot 10^{4}}{1,5^{2}}} \mathrm{~B} \approx 720 \mathrm{~B} .
$$

Одного аккумулятора мало. Для $n=2$ амплитуда равна

$$
U_{0}^{(2)}=24 \sqrt{1+\frac{0,81 \cdot 10^{4}}{4}} \mathrm{~B} \approx 1080 \mathrm{~B} .
$$

Двух аккумуляторов хватит.

П.Крюков да и вообще

$$
\frac{1}{2^{k}+1}+\frac{1}{2^{k}+2}+\ldots+\frac{1}{2^{k+1}}>\frac{2^{k}}{2^{k+1}}=\frac{1}{2},
$$

поскольку в левой части $2^{k}$ слагаемых и все они, кроме одного, больше $\frac{1}{2^{k+1}}$, а оставшееся ему равно. Поэтому

$$
1+\frac{1}{2}+\ldots+\frac{1}{2^{m}}>1+\frac{m}{2},
$$

т.е. сумма в левой части может быть сколь угодно большой.

Некоторым юным кружковцам этот факт кажется естественным: мы же можем брать сколько хотим слагаемых, конечно же, сумму можем увеличивать, как хотим. И следом идет нечто удивительное: сколько слагаемых в сумме $1+\frac{1}{2}+\frac{1}{4}+\frac{1}{8}+\ldots+\frac{1}{2^{n}}$ ни бери, результат будет меньше 2. Оказывается, зависит от того, какие слагаемые!

Когда я был школьником, меня удивила такая сюрреалистичная задача.

Задача 1. Резинка длиной один метр одним концом целпяется за вбитый гвоздь, а другой конеи, держит Андрей. На приби том коние сидит жук, который начинает ползти к Андрею со скоростью 1 cм/мин. Каждую минуту Андрей растягивает 
резинку еще на один метр. Доползет ли когда-то жук до Андрея?

На первый взгляд кажется, что каждую минуту оставшаяся жуку дистанция увеличивается на 99 сантиметров, поэтому как же он доползет? Но на самом деле жук ведь отдаляется от гвоздя вместе с растяжением резинки. Поэтому важно, что жук проползает не по 1 см каждую минуту, а сначала $\frac{1}{100}$ от всей длины, потом $\frac{1}{200}$, потом $\frac{1}{300}$ и т.д. Из расходимости гармонического ряда следует, что когда-то эта сумма станет больше 1.

Менее тривиальным оказывается следующий факт.

Теорема 2. Сумма $\frac{1}{4}+\frac{1}{9}+\ldots+\frac{1}{n^{2}}$ ограничена (числом, не зависящим от $n$ ).

Доказательство. Будем доказывать, что

$$
\frac{1}{4}+\frac{1}{9}+\ldots+\frac{1}{n^{2}}<\frac{3}{4} \text {. }
$$

Это неравенство следует из того, что

$$
\begin{array}{r}
\frac{1}{3^{2}}+\frac{1}{4^{2}}+\ldots \frac{1}{n^{2}}<\frac{1}{2 \cdot 3}+\frac{1}{3 \cdot 4}+\ldots+\frac{1}{(n-1) n}= \\
=\left(\frac{1}{2}-\frac{1}{3}\right)+\left(\frac{1}{3}-\frac{1}{4}\right)+\ldots+\left(\frac{1}{n-1}-\frac{1}{n}\right)= \\
=\frac{1}{2}-\frac{1}{n}<\frac{1}{2}
\end{array}
$$

Неравенство (1) позволяет доказать следующие изящное утверждение.

Теорема 3. Среди чисел от 1 до $2^{n+1}$ хотя бы $n$ простых.

Доказательство. Предположим, среди чисел от 1 до $2^{n+1}$ ровно $k$ простых чисел. Назовем число свободным от квадратов, если оно не делится ни на какой точный квадрат, больший 1.

Обозначим через $M$ количество свободных от квадратов чисел от 1 до $2^{n+1}$.

Лемма 1. $M \leq 2^{k}$.

Доказательство. Заметим, что в разложении каждого свободного от квадратов числа, меньшего $2^{n+1}$, входят только простые, меньшие $2^{n+1}$, причем в степени не выше первой. Поэтому каждое свободное от квадратов число определяется подмно- жеством своих простых делителей. Подмножеств из $k$ элементов, как мы знаем, $2^{k}$.

Лемма 2. $M>2^{n-1}$.

Доказательство. Оценим сверху количество чисел, несвободных от квадратов. Не более четверти всех чисел делится на 4 , не более девятой части делится на 9 и т.д. Таким образом, количество чисел, несвободных от квадратов, не более чем $\frac{2^{n+1}}{2^{2}}+\frac{2^{n+1}}{3^{2}}+\ldots+\frac{2^{n+1}}{\left(2^{n+1}\right)^{2}}$, что в свою оче-

$$
2^{n+1}\left(\frac{1}{2^{2}}+\ldots+\frac{1}{\left(2^{n+1}\right)^{2}}\right)<2^{n+1} \cdot \frac{3}{4}=3 \cdot 2^{n-1} .
$$

А тогда свободных от квадратов чисел больше $2^{n-1}$.

Неравенство $k>n-1$ легко получается из этих двух лемм, что и завершает доказательство теоремы 3.

Отметим, что теорема 3 следует из знаменитого постулата Бертрана, но нам удалось дать достаточно простое доказательство.

Отдельный интерес вызывает следующее утверждение.

Теорема 4. Если $p_{i}-i$-e по счету

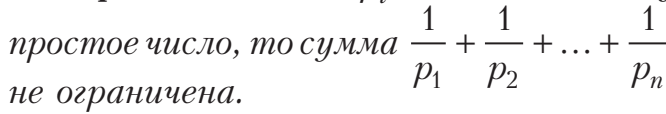

Отложив доказательство теоремы, решим с ее помощью следующую задачу задачу М2548 «Задачника «Кванта».

Задача 2. Натуральное число $п$ называется избыточным, если сумма всех его собственных делителей (т.е. натуральных делителей, отличных от самого n) больше n. Докажите, что для любого натурального $N$ существует $N$ подряд идущих избыточных чисел.

Лемма. Можно подобрать $k$ групп попарно различных простых чисел так, что сумма обратных величин к числам каждой группь больше 1.

Доказательство. Будем доказывать это утверждение индукцией по $k$.

База: $k=0$.

Переход: $k \rightarrow k+1$. Предположим, набрали $k$ групп и все выбранные простые числа меньше натурального $M$. Тогда за- 
метим, что сумма обратных к простым, большим $M$, неограничена, как и сумма обратных ко всем простым (поскольку сумма обратных к простым, не превосходящим $M$, конечная). Поэтому можно выбрать несколько простых, больших $M$, сумма обратных величин к которым больше 1. Таким образом, мы набрали еще одну группу. Лемма доказана.

Перейдем к решению задачи. Выберем $N$ групп простых чисел, о которых говорится в лемме. Теперь нам надо добиться того, чтобы $a$, первое из последовательных чисел, делилось на простые первой группы, второе - делилось на числа из второй группы, третье - на числа из третьей группы и т.д. Это будет выполнено, если $a \equiv 0\left(\bmod p_{1, i}\right), \quad a \equiv-1\left(\bmod p_{2, i}\right), \ldots$ $\ldots, \quad a \equiv-N+1\left(\bmod p_{N, i}\right)$, где $p_{l, i}$ пробегает простые числа из группы номер $l$. Каждое из этих требований - это сравнение по модулю простого числа. А китайская теорема об остатках гарантирует, что такая система по модулям различных простых разрешима. Но если число делится на все простые из какой-то группы, то оно избыточное. Действительно, если число $b$ делится на простые из группы номер $l$, у него уже есть собственные делители $\frac{b}{p_{l, 1}}, \frac{b}{p_{l, 2}}, \ldots$, а сумма этих делителей уже больше $b$. Таким образом, каждое из чисел $a, a+1, \ldots, a+N-1$ избыточно. Задача решена.

Теперь давайте докажем теорему 4. Начнем с нескольких вспомогательных утверждений.

Утверждение 1. Имеет место следуюшее неравенство:

$$
\begin{aligned}
\prod_{i=1}^{N}\left(1+\frac{1}{p_{i}}+\frac{1}{p_{i}^{2}}+\ldots\right. & \left.+\frac{1}{p_{i}^{N}}\right)> \\
& >1+\frac{1}{2}+\frac{1}{3}+\ldots+\frac{1}{N} .
\end{aligned}
$$

Доказательство. Если раскрыть скобки, то получится много слагаемых вида $\frac{1}{p_{1}^{\alpha_{1}} p_{2}^{\alpha_{2}} \ldots p_{N}^{\alpha_{n}}}$. Заметим, что каждое число $\frac{1}{k}$, где $k \leq N$, получится, потому что у любого такого $k$ в разложении на простые множители встречаются только простые, не большие $N$ и не более чем в $N$-й степени.

Утверждение 2. Для любого $\varepsilon>0$ существует такое $m$, что выполнено неравенство

$$
\left(1-\frac{1}{p_{1}}\right)\left(1-\frac{1}{p_{2}}\right) \ldots\left(1-\frac{1}{p_{m}}\right)<\varepsilon .
$$

Доказательство. Увеличим левую часть формулы (2), заменив каждую сумму геометрической прогрессии на сумму бесконечной, и получим

$$
\prod_{i=1}^{N} \frac{1}{1-\frac{1}{p_{i}}}>1+\frac{1}{2}+\frac{1}{3}+\ldots+\frac{1}{N} .
$$

Перевернем дроби в обеих частях со сменой знака:

$$
\prod_{i=1}^{N}\left(1-\frac{1}{p_{i}}\right)<\frac{1}{1+\frac{1}{2}+\frac{1}{3}+\ldots+\frac{1}{N}}
$$

Выберем такое $N$, для которого $1+\frac{1}{2}+\frac{1}{3}+\frac{1}{N}>\frac{1}{\varepsilon}$, и тем самым завершим доказательство утверждения.

Утверждение 3. Для любого С существует такое n, ито выполнено неравенство

$$
\left(1+\frac{1}{p_{1}}\right)\left(1+\frac{1}{p_{2}}\right) \ldots\left(1+\frac{1}{p_{n}}\right)>C .
$$

Доказательство. Предположим, что для любого $n$ выполнено

$$
\left(1+\frac{1}{p_{1}}\right)\left(1+\frac{1}{p_{2}}\right) \ldots\left(1+\frac{1}{p_{n}}\right) \leq C .
$$

Выберем такое $n$, для которого

$$
\left(1-\frac{1}{p_{1}}\right)\left(1-\frac{1}{p_{2}}\right) \ldots\left(1-\frac{1}{p_{n}}\right)<\frac{1}{2 C} .
$$

По утверждению (2) такое $n$ существует. Перемножим (6) и (5):

$$
\left(1-\frac{1}{p_{1}^{2}}\right)\left(1-\frac{1}{p_{2}^{2}}\right) \ldots\left(1-\frac{1}{p_{n}^{2}}\right)<\frac{1}{2} .
$$

Уменьшим левую часть, добавив множите- 
ли $\left(1-\frac{1}{k^{2}}\right)$ для составных $k<p_{n}$. После чего преобразуем каждую скобку $\left(1-\frac{1}{k^{2}}\right)$ как $\frac{(k-1)(k+1)}{k^{2}}$ и получим

$$
\frac{1 \cdot 3}{2^{2}} \cdot \frac{2 \cdot 4}{3^{2}} \cdot \frac{3 \cdot 5}{4^{2}} \cdot \ldots \cdot \frac{\left(p_{n}+1\right)\left(p_{n}-1\right)}{p_{n}^{2}}<\frac{1}{2} .
$$

Но нетрудно видеть, что левая часть последнего неравенства равна $\frac{p_{n}+1}{2 p_{n}}$. Очевидно, это не меньше $\frac{1}{2}$.

Поймем, как из этого следует теорема 4. Предположим, что для всех $n$ выполнено

$$
\frac{1}{p_{1}}+\frac{1}{p_{2}}+\ldots+\frac{1}{p_{n}}<C .
$$

Возведем число $e$ в степень обеих частей:

$$
e^{\frac{1}{p_{1}}+\ldots+\frac{1}{p_{n}}}=e^{\frac{1}{p_{1}}} \cdot e^{\frac{1}{p_{2}}} \cdot \ldots \cdot e^{\frac{1}{p_{n}}}<e^{C} .
$$

Как известно, $e^{x} \geq x+1$ для всех $x \geq 0$. Применим это к левой части (8) и получим

$$
\left(1+\frac{1}{p_{1}}\right)\left(1+\frac{1}{p_{2}}\right) \ldots\left(1+\frac{1}{p_{n}}\right)<e^{C} .
$$

Но по утверждению 3 произведение $\left(1+\frac{1}{p_{1}}\right)\left(1+\frac{1}{p_{2}}\right) \ldots\left(1+\frac{1}{p_{n}}\right)$ при некотором $n$ станет больше $e^{C}$. Противоречие.

Известно, что количество натуральных чисел, меньших $n$ и взаимно простых с $n$, вычисляется как

$$
\varphi(n)=n\left(1-\frac{1}{p_{1}}\right)\left(1-\frac{1}{p_{2}}\right) \ldots\left(1-\frac{1}{p_{m}}\right),
$$

где $p_{1}, p_{2}, \ldots, p_{m}$ - все различные простые делители $n$. Утверждение 2 означает, что бывают $n$, для которых $\frac{\varphi(n)}{n}<\varepsilon$ (при этом все $p_{i}$ могут быть достаточно большими). Это поможет разобрать интересную задачу, придуманную по мотивам одной старой задачи Ленинградской олимпады.

Задача 3. Дан правильный п-угольник. Любое подмножество его вершин назовем шаблоном. Назовем шаблон маленьким, если в нем меньше $\frac{1}{100}$ от общего числа вершин. Могло ли при каком-то п ока- заться, что любые 100 шаблонов, получаюшихся из некоторого маленького шаблона поворотом, имеют обшую вершину?

Решение. Подберем $n$ в виде произведения нескольких различных простых $p_{1} p_{2} \ldots p_{k}$, каждое из которых больше 100, так , чтобы $\frac{\varphi(n)}{n}<\frac{1}{100}$. Выберем требуемый шаблон - все вершины, номера которых взаимно просты с $n$. Тогда в шаблон войдут менее $\frac{n}{100}$ вершин. Докажем, что любые 100 шаблонов, полученных из данного поворотами, пересекаются.

Действительно, предположим, что это повороты на $a_{1}, a_{2}, \ldots, a_{100}$. Для каждого простого $p_{i}$ подберем остаток $r_{i}$, не сравнимый с $a_{1}, a_{2}, \ldots, a_{100}$ по модулю $p_{i}$. Такой остаток существует, поскольку $p_{i}>$ $>$ 100. А теперь подберем остаток $a$ при делении на $n$, сравнимый с $r_{i}$ по модулю $p_{i}$ для всех $i$. Такой остаток $a$ найдется по китайской теореме об остатках.

Вершина с номером $a$ окажется во всех шаблонах. Действительно, для любого $j$ от 1 до 100 выполнено $a-a_{j} \equiv r_{i}-a_{j} \not \equiv 0$ $\left(\bmod p_{i}\right)$. Значит, $a-a_{j}$ взаимно просто с $n$.

Желающие попрактиковаться могут попробовать свои силы в следующих задачах. Первые две из них скорее упражнения, последняя же задача по-настоящему сложная.

Задача 4. Петя утверждает, что начиная с некоторого момента каждое следующее простое число больше предыдущего хотя бы в $\left(1+10^{-10}\right)$ раз. Докажите, что он не прав.

Задача 5. Обозначим $\sigma(n)$ сумму делителей числа $n$. Дано число $x>1$. Докажите, что существует такое целое $n$, что

$$
x<\frac{\sigma(n)}{n}<x+10^{-6} .
$$

Задача 6. Натуральное $n$ назовем интересным, если $\frac{\varphi(n)}{n}<\frac{1}{100}$. Докажите, что существует 1000 подряд идущих интересных чисел.

Задача 7. Множество вычетов $A$ по модулю $n$ назовем свободным от произведений, если $x y \not \equiv z(\bmod n)$ для всех $x, y, z \in A$ (необязательно различных). Наибольшее количество элементов в множестве, свободном от произведений, обозначим $f(n)$. Существуют ли такие $n$, для которых $f(n)>\frac{n}{2}$ ? 